\title{
Fabrication and Characterization of Low-Cost Poly(Vinyl Alcohol) Composite Membrane for Low Temperature Fuel Cell Application
}

\author{
Ruhilin Nasser, Siti Khadijah Hubadillah, Mohd Hafiz Dzarfan Othman*, Arif Akmal \\ Mohamed Hassan \\ Advanced Membrane Technology Research Centre, Faculty of Chemical and Energy \\ Engineering, Universiti Teknologi Malaysia, 81310 UTM Johor Bahru, Johor, \\ Malaysia
}

Submitted: 2/5/2018. Revised edition: 23/5/2018. Accepted: 27/5/2018. Published online: 4/6/2018

\begin{abstract}
The urge to find alternative sources of energy is crucial as the source of fossil fuel shows a high number of depletion over the year. Compared to other alternatives sources, fuel cell is high at rank as it generates no harmful gases to the surrounding and high in efficiency. The performance of this fuel cell is affected by several factors and one of it is the permeability of proton exchange membrane (PEM). Nafion ${ }^{\circledR}$ is known to be used as the PEM in fuel cells, however due to its high price, polyvinyl alcohol membrane was selected in this study to substitute the Nafion ${ }^{\circledR}$ as it was low in price and excellent in chemical and mechanical strength. Poly (vinyl alcohol) composite membrane was prepared and crosslinked with sulfosuccinic acid (SSA). To further increase the proton conductivity of the membrane, graphene oxide (GO) with 1, 2 and 3 weight percentage was incorporated into the polymer membrane. All the membranes were characterized by using Fourier transform infrared (FTIR) spectroscopy, scanning electron microscopy (SEM), water uptake measurement, contact angle, ion exchange capacity and proton conductivity respectively. Synthesized membranes show low water uptake and contact angle as GO loading was increased. IEC value and water swelling were found to be increased with increasing of GO loading. The proton conductivity of the membrane increases as more GO was incorporated into PVA-SSA and achieved its highest conductivity at $0.020746 \mathrm{~S} \mathrm{~cm}^{-1}$ with $2 \mathrm{wt} . \%$ of GO incorporation.
\end{abstract}

Keywords: Proton Exchange Membrane (PEM), Poly (vinyl alcohol), Graphene Oxide, Composite Membrane, Proton Conductivity

\subsection{INTRODUCTION}

For the past previous years, the demand of fossil fuel for energy supply is always at the highest of rank and exclusively use to generate power due to its high availability and low in price.

However, as time goes by the world is now facing the shortage of fossil fuel and the urge to find other alternative sources of energy is crucial. In addition to climate changes such as global warming and other environmental effects, attraction towards sustainable energy or so-called as green energy has been inclined. Renewable energy such as biomass is one of the promising energy supply that regarded as a carbon neutral.

As an another upshot to these matter, fuel cell that uses high value metal catalyst traditionally to generate power has drawn various attention as alternative energy sources. In fact, fuel cell surprisingly dominates other types of energy generators as no emission of environmental pollution gases such as sulphur dioxide, carbon dioxide and 
higher efficiency. In spite of that, high cost and high mass generation are the only drawbacks of these kind of energy sources. To define a fuel cell, it is a device that convert chemical energy to electricity producing only water and heat as by-product [2]. The reactions in the fuel cell can be displayed as following,

Anode reaction $\quad: \mathrm{H}_{2} \rightarrow 2 \mathrm{H}^{+}+2 \mathrm{e}^{-}$ Cathode reaction : $1 / 2 \mathrm{O}_{2}+2 \mathrm{H}^{+}+2 \mathrm{e} \rightarrow$ $\mathrm{H}_{2} \mathrm{O}$

Overall reaction $\quad: \mathrm{H}_{2}+1 / 2 \mathrm{O}_{2} \rightarrow$

$\mathrm{H}_{2} \mathrm{O}+$ Electrical Energy + Heat energy

Fuel cell is generally categorized accordingly to the type of fuel, operating condition like temperature and nature of electrolyte. Fuel cell that operated at low temperature are namely proton exchange membrane fuel cell, (PEMFC), direct methanol fuel cell (DMFC) and Microbial fuel cell (MFC). Generally, the working principle of these three fuel cells are the same. The hydrogen ions at the anode will diffuse to the cathode through the electrolyte membrane that will generate electricity and only water as the byproduct. The key component of these fuel cells is the polymer electrolyte membrane (PEM). The PEM holds three roles in this system, first as the proton charge carrier, second to separate the reactant gases and third as an electronic insulator [3]. A better PEM should possess high proton conductivity, low fuel permeability, high oxidative stability, good mechanical strength and most importantly low cost of fabrication.

Perflourinated membranes, Nafion ${ }^{\circledR}$ membrane has been chosen as the standard for PEM due to its high thermal and chemical stability and high proton conductivity and the most popular to be used as PEM. However, Nation ${ }^{\circledR}$ is high in cost (US \$ 800-2,000 $\mathrm{m}^{-2}$ ) [4] and also faces several short comes such as limited working in low temperature and high methanol cross over. Thus, there is an urgent need to develop a low cost membrane to create an affordable membrane to be used in the low temperature fuel cells system.

Nowadays, poly (vinyl alcohol) (PVA) based polymer electrolyte membranes have attractf many interest of researchers to be used in various fields including fuel cells, proton exchange and pervaporation. This low cost PVA inorganic composite membranes show excellent in dimensional, thermal and mechanical stability, good hydrophilic and electrochemical properties, all of which are crucial for good proton exchange membranes [5]. Nevertheless, compared to Nafion ${ }^{\circledR}$, PVA is a poor proton conductor membrane as it does not possess any negative charged ions such as carboxylic and sulfonic acid groups [6, 12-13]. A good membrane should high in proton conductivity as well as high in ion exchange capacity. However, high IEC will cause the membrane to experience excessive swelling. As a result, these membranes will lose their mechanical stability and not practicable to be used in fuel cell.

As an upshot to these matters, techniques of ionic crosslinking of acid based blend PEM and covalent cross linking PEM were developed. Covalent cross linking of membrane can give better impact strength and resistance to load deformation without yielding and loosening by which the loss of sulfonated polymer can be reduced. In this present study, sulfosuccinic acid (SSA) was used as the cross linker to provide main source of proton conduction. But still with this combination PVA-SSA it still shows relatively low chemical stability and low proton conductivity as compared to Nafion.

Thus, to overcome this drawback, incorporation of inorganic-organic composite membrane were developed as the new PEM. This composite 
system can also cause an increase in chemical and mechanical stability. Incorporation of an ion conductive filler to the membrane also has the ability to improve its conductivity. Graphene oxide (GO) is a two-dimensional single-layered homologue of graphene containing various oxygen functional groups (e.g., hydroxyl, epoxide, carbonyl, carboxyl) that has wide application in supercapacitors, photovoltaic devices, biosensors, and nanocomposite.

The incorporation of GO into a polymer matrix capable to improves many of its physical and chemical properties, including ion conductivity and mechanical strength. It is anticipated that the addition of GO into the polymer matrix, PVA-SSA could improve the conductivity of polymer electrolyte.

Different loading of GO was used to investigate the effect toward membrane's proton conductivity [5]. To overcome this deficiency, incorporation of inorganic-organic composite membrane were developed as the new PEM [3]. Some inorganic material like silicon oxide, titanium oxide and zirconium phosphate have been introduced to balance good proton conductivity and low fuel permeability [2]. Inorganic zirconium phosphate especially is very popular for its ion exchange capacity [1-2]. Eventually, the objective of this study was to examine the effect of GO addition on the properties of the PVA membranes crosslinked with sulfonic acid group and to evaluate its potential as a feasible PEM for low temperature fuel cell and comparing it with the standard membrane, Nafion and PVA-SSA-ZrP.

\subsection{METHODS}

\subsection{Materials}

Palm oil (cooking oil) as diluent Poly (vinyl alcohol) (99\% hydrolysed, MW=145 000), sulfosuccinic acid (SSA) (70 wt. \% aqueous solution), zirconyl chloride octahydrate (reagent grade, $98 \%)$ and phosphoric acid $(85 \%$ hydrolysed) were purchased from Sigma Aldrich and used as the polymer matrix, cross linking agent and synthesis process of zirconium phosphate, respectively.

\subsection{Synthesis of Zirconium Phosphate}

Zirconium phosphate (ZP) was synthesised by using reflux method. 10 $\mathrm{g}$ of zirconyl chloride octahydrate was mixed with $6 \mathrm{M}$ of $100 \mathrm{ml}$ of phosphoric acid and heated up to $110^{\circ} \mathrm{C}$ in oil bath. ZP produced was further treated by adding de-ionised water and centrifuged to obtain the desired ZP.

\subsection{Preparation of Composite Membrane}

The GO-dispersed PVA-SSA membranes were prepared by using mixing and casting method. The control membrane, PVA-SSA membrane (10 wt. \% PVA with 5 wt. \% SSA) was fabricated by dissolving the $10 \mathrm{~g}$ of PVA in $90 \mathrm{~mL}$ of distilled water at 90 ${ }^{\circ} \mathrm{C}$ for at least $6 \mathrm{~h}$. As the PVA solution cooled to room temperature, 5 wt. \% SSA was added. Then various loading of GO (1.0, 2.0, 3.0 wt. \%) and 5 wt. \% of $\mathrm{ZrP}$ were added. The GO was obtained from our collaborator in Universiti Pendidikan Sultan Idris (UPSI), Perak which synthesized the GO using Hammer's method. 
The mixtures PVA-SSA-GO $(1,2$ and 3 wt. \%) and PVA-SSA-ZrP (5 wt. \%) were vigorously stirred at room temperature for $24 \mathrm{~h}$. Then, the solutions were casted onto a Plexiglas plate. The cast membranes were allowed to dry at $60{ }^{\circ} \mathrm{C}$ in a forced convection oven. After it sufficiently dried, it was heated for $1 \mathrm{~h}$ in a thermoset oven at desired temperatures, $120{ }^{\circ} \mathrm{C}$.

\subsection{Membrane Characterization}

The presence of various functional groups in polymer was determined by diffusing reflectance Fourier transform infra-red (FT-IR) spectroscopy. FT-IR spectra was measured at room temperature and represent of about 200 scans at a resolution of $2 \mathrm{~cm}^{-1}$ [7] operating in the wave number range of $500-4000 \mathrm{~cm}^{-1}$. It was also used to examine the interaction of raw material of the composite membrane, PVASSA-GO.

The surface and cross sectional features of the PVA-SSA as well as the composite membrane's morphologies were observed by using Scanning Electron Microscopy (SEM).

\subsection{Water Uptake and Swelling Measurement}

As being discussed by Yee et al., (2013), membranes that have been soaked were weighed and measured to determine wet weight and wet length. Then the samples were dried overnight and again its dry weight and length were measured [8]. The water uptake and swelling were calculated by the equations below,

Water Uptake $=[$ (wet weight-dry weight)/ dry weight] x $100 \%$

Water Swelling $=[($ wet length-dry length)/ dry length] x $100 \%$

\subsection{Ion Exchange Capacity}

Titration method was used to determine the ion-exchange capacity (IEC). Each membrane sample was soaked in $50 \mathrm{~mL}$ of $1 \mathrm{M}$ hydrochloric acid for an hour. Then the membranes was washed with deionized water. After that, it was soaked in $50 \mathrm{~mL}$ of I M of sodium chloride aqueous solution overnight to exchange protons with sodium ions. The ion-exchanged solution was titrated with $0.001 \mathrm{M}$ sodium hydroxide solution. The IEC value was then determined using the following equation.

$\mathrm{IEC}=\left[0.01\left(\mathrm{~V}_{\mathrm{o}, \mathrm{NaOH}}-\mathrm{V}_{\mathrm{E}, \mathrm{NaOH}}\right)\right] / \mathrm{W}_{\text {dry }}(3)$

where $\mathrm{V}_{\mathrm{o}, \mathrm{NaOH}}$ is the amount of $\mathrm{NaOH}$ solution before titration, $\mathrm{V}_{\mathrm{E}, \mathrm{NaOH}}$ is the amount of $\mathrm{NaOH}$ solution after titration, $\mathrm{W}_{\text {dry }}$ is the weight of the dry membrane.

\subsection{Proton Conductivity Measurement}

Most important property of this fuel cell is its proton conductivity. The impedance of the membranes was determined by using a four-point probe cell connected with an impedance/gain phase analyser, Autolab PGSTAT, Methrohm Autolab. Prior to each analysis, the membranes were cut into strips and mounted in the cell that placed in a thermo-humidity controlled chamber. Then, an AC current was allowed to pass through two outer electrodes and the AC potential difference between the two inner electrodes was the conductance of the samples. The measurement of the proton conductivity, was calculated by using following formula,

$$
\sigma=\frac{l}{R S}
$$

where $\sigma$ was the proton conductivity $(\mathrm{S} / \mathrm{cm}), \mathrm{R}$ the ohmic resistance, $l$ the 
distance between the counter electrode and the counter electrode $(\mathrm{cm})$ and $\mathrm{S}$ the cross-sectional area of the membrane sample $\left(\mathrm{cm}^{2}\right)$

\subsection{RESULTS AND DISCUSSION}

\subsection{Characterization of Composite Membrane}

The morphologies of the membrane and dispersion of GO into the PVA-SSA were analysed using SEM technique. Figure 1 (a, c and e) shows the surface morphologies, whereas Figure 1 (b, d and $f$ ) were the cross-sections of the membranes. The PVA-SSA membrane appeared to be dense and compact. As $\mathrm{GO}$ is introduced into the PVA-SSA polymer matrix, the membrane becomes rough and displays obvious wrinkles. The membrane with 2 wt. \% of GO displays better dispersion along the surface and cross-section of the membrane. This is probably because of $(-\mathrm{COOH})$ groups on the GO might help the dispersion of $\mathrm{GO}$ by generating attractive interaction with $(-\mathrm{OH})$ groups on the PVA-SSA polymer matrix and thus improving the interfacial compatibility. The favourable dispersion of GO would provide more continuous pathways for proton migration which expected would result to higher proton conductivity. However, as the loading of GO is further increased to $3 \mathrm{wt}$. $\%$, an aggregations of GO appear as irregular masses and not dispersed uniformly.

The FT-IR spectra of PVA-SSA and PVA-SSA-GO films were shown in Figure 2 (a) and (b) respectively. In the PVA-SSA, the peak exists at 2925, 1407 and 843 and $1033 \mathrm{~cm} \mathrm{-1} \mathrm{are}$ attributed to the $\mathrm{C}-\mathrm{H}$ stretching, $\mathrm{C}-\mathrm{H}$ bending, $\mathrm{C}-\mathrm{O}$ bending and $\mathrm{C}-\mathrm{O}$ stretching of PVA, respectively. The broad high absorption peak at $3352 \mathrm{~cm}$ -1 is assumed to arise from the $\mathrm{O}-\mathrm{H}$ stretching frequencies of PVA and water hydroxyl groups. The band at $1785 \mathrm{~cm}-1$ shows the carbonyl functional groups. The changes of the characteristic spectral peaks reflect the chemical interactions, when two or more substances are mixed. Meanwhile, in the spectrum of the PVA-SSA-GO (3) composite film. The GO characteristic peaks present at $3430 \mathrm{~cm}-$ 1 O-H stretching, $1710 \mathrm{~cm}^{-1}$ ( CO stretching), $1641 \mathrm{~cm}^{-1}$ (CC stretching), $1407 \mathrm{~cm}^{-1}$ (OH bending), $1211 \mathrm{~cm}^{-1}$ (CO epoxy bending) and $1033 \mathrm{~cm}^{-1}$ for (CO alkoxy stretching) respectively.
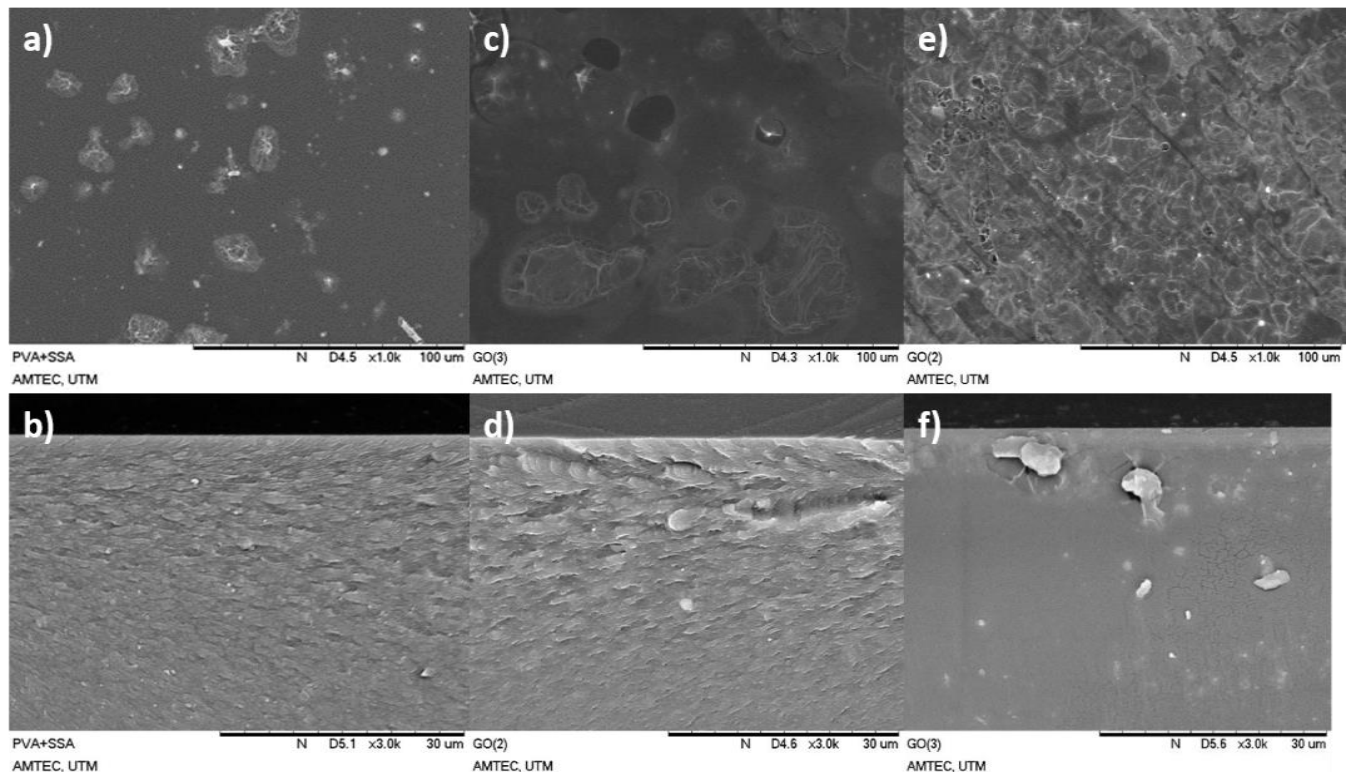

Figure 1 Surface area and cross section SEM images of (a-b) PVA-SSA, (c-d) PVA-SSA$\mathrm{GO}(2)$ and (e-f) PVA-SSA-GO(3) 
The peak of O-H increases from 3352 $\mathrm{cm}^{-1}$ on PVA-SSA to $3430 \mathrm{~cm}^{-1}$ after incorporation of GO. This indicates there are existence of bonding interactions between the hydroxyl groups. Whereas, the carbonyl groups peak decreases from $1785 \mathrm{~cm}^{-}{ }^{1}$ in PVASSA to $1710 \mathrm{~cm}^{-1}$ as GO is introduced to the polymer membrane.

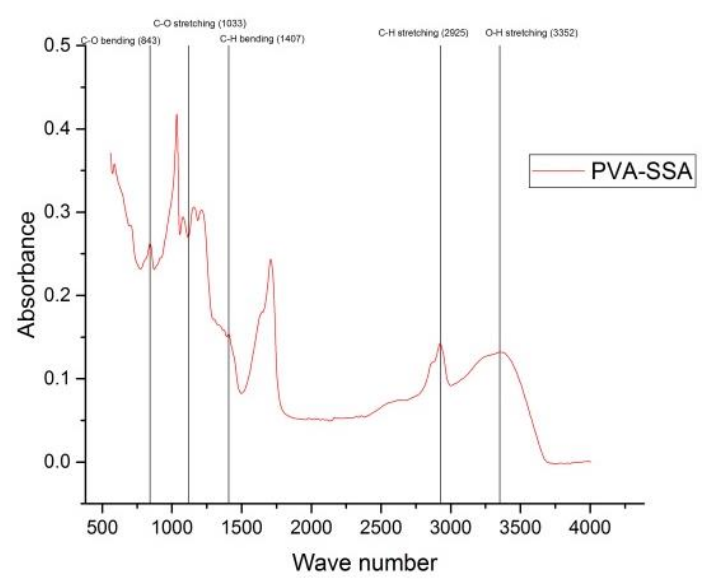

Figure 2 (a) FTIR spectrum of PVA -SSA

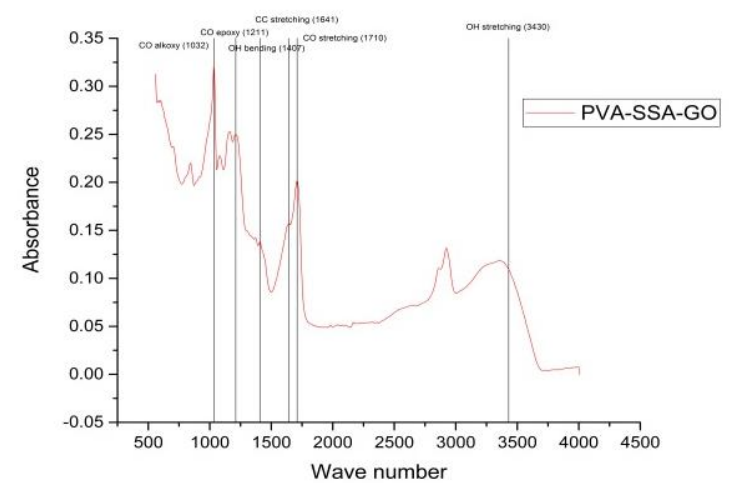

Figure 2 (b) FTIR spectrum of PVA-SSAGO

\subsection{Water Uptake and Contact Angle}

Water in PEMs plays an important role in proton conduction because water molecules can facilitate the transport of proton by providing proton medium (free water), forming hopping hydrogen-bonded networks (bounded networks (bound water), and dissociating conducting groups (free water).The water uptake of PVA-SSA control membrane is around $17 \%$.
From Figure 3, it can be seen that with an increase of addition of hydrophilic $\mathrm{GO}$, it reduces the water uptake of the membrane. This is probably because of better interaction between $-\mathrm{COOH}$ groups on the $\mathrm{GO}$ and $-\mathrm{OH}$ groups in the PVA-SSA polymer matrix. The reduction in water uptake may also responsible from the slight loss of proton conductivity. Meanwhile, as $\mathrm{ZrP}$ was incorporated into the PVA-SSA, the water uptake also decreases but still higher than GO which is at $13.898 \%$. In other words, incorporation of GO reduces the water uptake of PVA-SSA more compared to $\mathrm{ZrP}$.

For contact angle, as the loading of GO is increased to the PVA-SSA polymer matrix, its contact angle decreases. This is explained by the fact of that introducing more $\mathrm{COOH}$ group into the membrane matrix leads to the increase of the membrane polarity and hydrophilicity of the membrane.

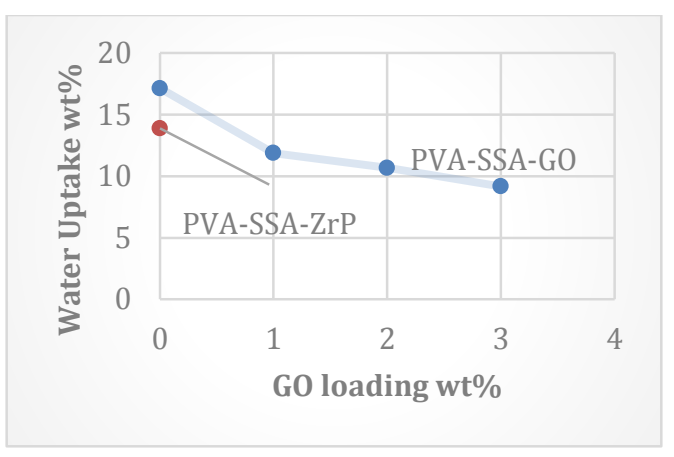

Figure 3 Water uptake measuremet of the membrane towards different loading of GO

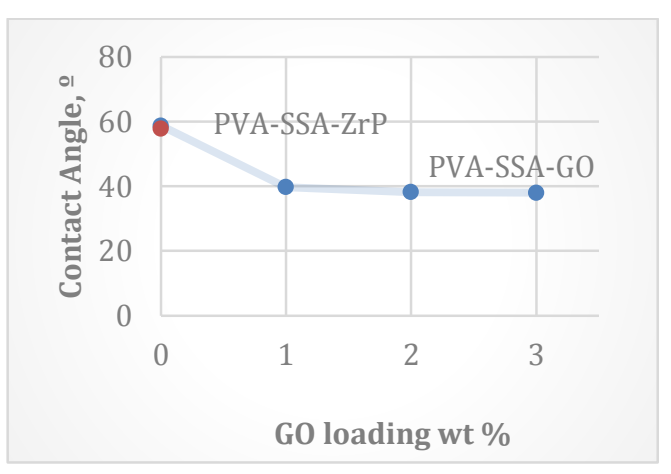

Figure 4 Contact angle of the membrane towards different loading of GO 


\subsection{Water Swelling}

Based on Figure 5, as more GO is incorporated into PVA-SSA polymer matrix, the swelling of the membrane increased. PVA is a hydrophilic polymers and GO can readily form a stable colloidal suspension in water [10]. Both hydroxyl and carboxyl groups in PVA-SSA-GO possess high affinity towards water molecules. The hydroxyl groups in the polymer matrix membrane have the ability to connect with water molecules through the formation of hydrogen bonds. However, as the GO loading is further increased to $3 \mathrm{wt}$. \%, it swelling decreases to $7.5 \%$. This may be because of low hydrogen bond created between PVA and water molecules. Meanwhile, for PVA-SSA$\mathrm{ZrP}$, the swelling of this membrane was low compared to other membranes.

Another remarkable reasons may be attributed to the structure of $\mathrm{ZrP}$ and GO that have nanoplatelets and nanosheets structure, respectively. Accordingly, the aqueous-phase separation capability of a layer-stacked GO I membrane structure can be significantly limited by its natural tendency to swell, that is, absorb water into the GO channel and form enlarged interlayer spacing [11].

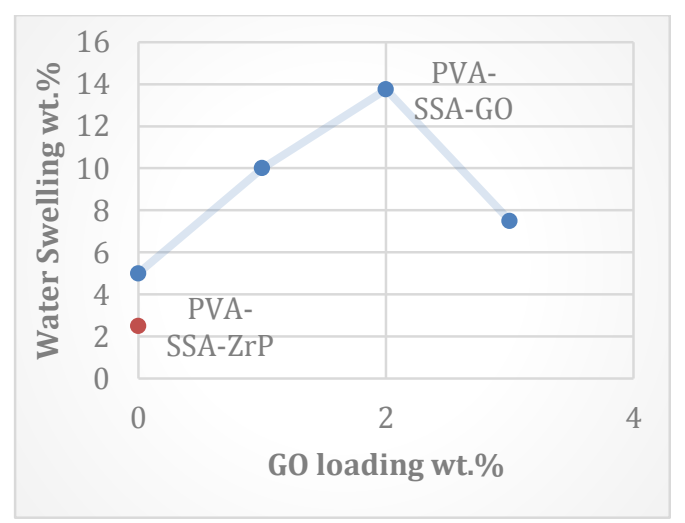

Figure 5 Water Swelling of Membrane

\subsection{Ion Exchange Capacity}

The presence of acidic sites, carboxyl on the basal planes of GO enables GO to exhibit capability of proton exchange. As shown in Figure 6, the IEC value of control membrane, PVA-SSA was 60.79 mmol g- ${ }^{1}$. By comparison, the PVA-SSA-GO (3) achieved the highest IEC value at $65.24 \mathrm{mmol} \mathrm{g}^{-}{ }^{1}$. Incorporation of $\mathrm{GO}$ gives an increase of IEC values, which is originated from the proton conducting groups $(-\mathrm{COOH})$ on GO. Elevating the $\mathrm{COOH}$ group that is $\mathrm{GO}$ content will further increased the IEC values. As the GO content increased from $1 \mathrm{wt} . \%$ to $2 \mathrm{wt} . \%$ and finally to $3 \mathrm{wt} \%$, the IEC value increased from $61.16 \mathrm{mmol} \mathrm{g}_{-}{ }^{1}$ to 61.79 mmol g- ${ }^{1}$ and further to $65.24 \mathrm{mmol} \mathrm{g-}{ }^{1}$. However, as for PVA-SSA-ZrP it possess the lowest IEC value, $59.97 \%$ due to absence of any acidic sites.

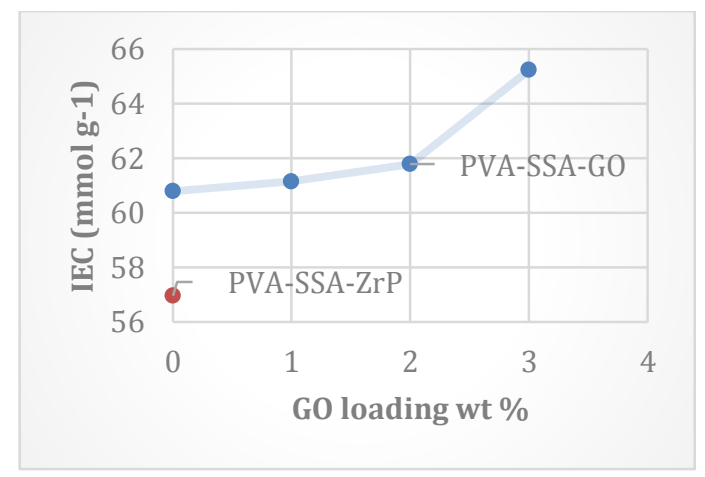

Figure 6 Ion Exchange Capacity of Membrane

\subsection{Proton Conductivity Measurement}

The proton conductivities of the membrane were determined by $\mathrm{AC}$ impedance analysis over the frequency of $10 \mathrm{~Hz}$ to $10 \mathrm{kHz}$ under a $10.0 \mu \mathrm{A}$. The proton released from the $\mathrm{COOH}$ groups on the basal plane of GO allows it to possess proton conductivity. Despite of the reduced water uptake that should slow down the transfer of 
protons via water, the conductivity increases as the loading of GO was increased. Such observation is reasonably attributed to two reasons. First, GO would donate sufficient proton hoping sites in the form $-\mathrm{COOH}$ as testified in by the increase in the IEC value. Then, better dispersion of GO that would provide more wide and longrange conducting pathways within the membrane.

Among the series of the GO dispersed PVA-SSA, the membrane with 2 wt.\% GO possess the highest proton conductivity, $0.020746 \mathrm{~S} \mathrm{~cm}^{-}$. A 4.18 fold increase in conductivity was recorded upon the incorporation of 2 wt. \% GO in a PVA-SSA membrane as compared to pure PVA-SSA membrane polymer matrix. The improvement can be justified by the increase in the number of ion-exchange groups ($\mathrm{COOH}$ group of GO), along with an increase in the interconnected transfer channels in the presence of GO. Whereas, as ZrP was added to PVASSA, the proton conductivity also increases up to $0.009353 \%$. However, this value was still low as compared to PVA-SSA-GO (2). Thus, it shows that $\mathrm{GO}$ is better than $\mathrm{ZrP}$ in terms of increasing the proton conductivity of membrane. Yet, the proton conductivity of the membrane PVA-SSA-GO (2) was still found to be less than commercial, Nafion 115 membrane, $0.0404 \mathrm{~S} \mathrm{~cm}^{-}$.

Then, as the loading of GO is increased to $3 \mathrm{wt} \%$, its conductivity started to reduce to $0.005959 \mathrm{~S} \mathrm{~cm}^{-}$. The conductivity decreases probably because of the decreased $\mathrm{GO}$ as the proton moiety and attributed to the 'blocking affect' of filler loading.

\subsection{CONCLUSION}

A series of composite membranes consist of polyvinyl alcohol (PVA), sulfosuccinic acid (SSA) as the cross linker agent and graphene oxide (GO) were prepared as proton conducting membrane for low temperature fuel cells application. These membranes also known as PEM were developed to produce a low cost, comparable and feasible PEM replacing the high cost Nafion membrane. The proton conductivity of the membrane was increased as GO was introduced to the PVA-SSA polymer matrix. GO loading of 2 wt. $\%$ into PVA-SSA polymer matrix shows the highest conductivity at $0.0207 \mathrm{~S} \mathrm{~cm}^{-1}$. This value was higher than PVA-SSA-ZrP and yet still low than the Nafion 115, which was 0.0404 $\mathrm{S} \mathrm{cm}^{-}{ }^{1}$.

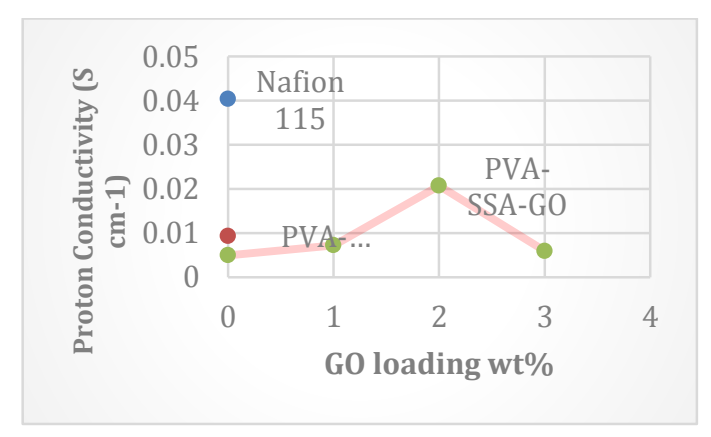

Figure 7 Proton Conductivity

\section{ACKNOWLEDGEMENTS}

The authors gratefully acknowledge financial support from Universiti Teknologi Malaysia under Research University Grant Tier 1R\&D Fund (Project number: Q.J130000.7746.4J309Q.J130000.254 6.12H25) and Flagship UTMShineTier 1 (Project number: Q.J130000.2546.16H40Q.J130000.244 6.03G29), and Nippon Sheet Glass Foundation for Materials Science and Engineering under Overseas Research Grant Scheme (Project number: Q.J130000.2446.03G29). The authors would also like to thank Research Management Centre, Universiti 
Teknologi Malaysia for the technical support.

\section{REFERENCES}

[1] K. Watanabe. 2008. Recent Developments in Microbial Fuel Cell Technologies for Sustainable Bioenergy. J. Biosci. Bioeng. 106: 528-536.

[2] M. Rikukawa and K. Sanui. 2000. Proton-conducting Polymer Electrolyte Membranes based on Hydrocarbon Polymers. Prog. Polym. Sci. 25: 1463-1502.

[3] S. J. Peighambardoust, S. Rowshanzamir, and M. Amjadi. 2010. Review of the Proton Exchange Membranes for Fuel Cell Applications. Int. J. Hydrogen Energy. 35: 9349-9384.

[4] L. Yu. Yu, H. M. Shen, and Z. L. Xu. 2009. PVDF-TiO2 Composite Hollow Fiber Ultrafiltration Membranes Prepared by TiO2 SolGel Method and Blending Method J. Appl. Phys. 113: 1763-1772.

[5] S. Khilari, S. Pandit, M. M. Ghangrekar, D. Pradhan, and D. Das. 2013. Graphene OxideImpregnated PVA-STA Composite Polymer Electrolyte Membrane Separator for Power Generation in a Single-Chambered Microbial Fuel Cell. Ind. Eng. Chem. Res. 52: 11597-11606

[6] Y. Wang and Y. Hsieh. 2010. Crosslinking of Polyvinyl Alcohol (PVA) Fibrous Membranes with Glutaraldehyde and PEG Diacylchloride. J Appl Polym Sci. 116: 3249-3255.

[7] Y. Kozawa, S. Suzuki, M. Miyayama, T. Okumiya, and E. Traversa. 2010. Proton Conducting Membranes Composed of Sulfonated Poly(etheretherketone) and Zirconium Phosphate Nanosheets for Fuel Cell
Applications. Solid State Ionics. 181: 5-7.

[8] R. Yee, K. Zhang, and B. Ladewig. 2013. The Effects of Sulfonated Poly(ether ether ketone) Ion Exchange Preparation Conditions on Membrane. Properties. Membranes (Basel). 3: 182-195.

[9] J. Rhim, H. Bum, C. Lee, J. Jun, D. Sik, and Y. Moo. 2004. Crosslinked Poly (Vinyl Alcohol) Membranes Containing Sulfonic Acid Group: Proton and Methanol Transport Through Membranes. J. Membrane Sci. 238: 143-151.

[10] V. A. Online 2014. Waterinduced Shape Memory Effect of Graphene Oxide Reinforced Polyvinyl Alcohol. J. Mater. Chem. A. 2: 2240-2249.

[11] S.Zheng, Q.Tu, J.J. Urban, S.Li and B. Mi. 2017. Swelling of Graphene Oxide Membranes in Aqueous Solution: Characterization of Interlayer Spacing and Insight into Water Transport Mechanisms. ACS Nano. 11: 6440-6450.

[12] N. N. N. Mustofar, J. Jaafar, M. Aziz, A. F. Ismail, M.A. Rahman, M. H. D. Othman, N. Yusof, W. N. W. Salleh, F. Aziz, M. S. Rosmi. 2017. Transport Behavior in Polymer-Inorganic Membrane: A Review. J. Applied Membrane Science \& Technology. 19: 43-56.

[13] S. Koonaphapdeelert, Z. Wu, K. Li. 2009. Thermogravimetric Analysis and Stability Test of Fluoroalkylsilanes Grafted on Alumina Hollow Fibre Membranes, J. Applied Membrane Science \& Technology. 10: 51-59. 